\title{
10.
}

\section{Bemerkungen über die kleinste Fläche innerhalb gegebener Grenzen*).}

(Vom Herrn Prof. H. F. Scherk in Kiel.)

1.

Bereits seit längerer Zeit hat die kleinste von gegebenen Linien begrenzte Fläche die Aufmerksamkeit der Mathematiker in einem hohen Grade, sowohl wegen ihrer eigenthümlichen Eigenschaften, als wegen der Schwierigkeiten erregt, welche sich einer genaueren Untersuchung, und einer durch continuirliche Bewegung entstandenen Construction derselben entgegensetzen. Nachdem Lag range die Frage zuerst angeregt, und die Gleichung der Fläche, in Form einer partiellen Differentialgleichung vom zweiten Grade, gefunden hatte, zeigte Meusnier, dals ihr auch noch eine andere merkwürdige Eigenschaft zukomme: der grö̊ste und der kleinste Krümmungshalbmesser sind nämlich in jedem ihrer Puncte gleich grofs, und entgegengesetzt. Auch fand Meusnier, zwar nicht das vollstïndige Integral der erwähnten Differentialgleichung, aber doch zwei partielle Fälle desselben : die Gleichungen zweier speciellen krummen Flächen nämlich, welche der allgemeinen Gleichung Genüge leisten; und zwar erstens, der Schraubenfläche, d. i. derjenigen Fläche, welche entsteht, wenn eine horizontale gerade Linie sich so bewegt, dafs sie stets durch eine verticale Gerade und durch eine Schraubenlinie geht, die sich um die OberHläche eines geraden Cylinders hinaufwindet, als dessen Axe die genannte Verticale angesehen wird; und zweitens, derjenigen Fläche, welche durch Umdrehung der Kettenlinie um eine horizontale, sie nicht schneidende, Axe erzeugt wird **). Das allgemeine Integral der Gleichung unserer Fläche fanden zuerst Monge und Legendre, aber in einer so verwickelten Form, dafs

*) Diese Abhandlung ist im September 1833 der Königl. Akad. der Wissęnsch. zu Copenhagen vorgelegt worden.

* worden von $G$ oldschinidt: Determinatio superficiei minimae, rotatione cumvae data duo puncta jungentis circa datum axem ortae: Goettingae. 1831.

Crelle's Journal d. M. Bd. XIII. Hft. 3. 
186 10. Scherk, Bemerkungen über die kleinste Fläche innerhalbigegebener Grenzen.

man bis jetzt von dem Integrale fast noch keinen Gebrauch gemacht hat. So urtheilt auch Poiss on, bei Gelegenheit der Ankündigung einer neuen von ihm angestellten Untersuchung über diesen Gegenstand: einer Untersuchung übrigens, der man mit der gespanntesten Erwartung entgegen sehen muls: "Malheureusement on ne sauroit tirer aucune partie de ceite intégrale, qui se trouve compliquée de quantités imaginaires, et exprimée par le système de trois équations entre deux variables auxiliaires et les coordonnées courantes de la surface." (S. gegenw. Journal, VIII. Bd. pag. 361.). Eine neue, specielle Fläche hat Monge nicht angegeben; und da die Untersuchung über die characteristischen Linien der Flïche ihn darauf fuihrte, dafs dieselben in einem Puncte beständen, (ein Umstand, der darauf hinzudeuten scheint, dafs nicht alle Flächen als Einhüllungsflächen betrachtet werden können): so gelang es ihm auch nicht, die Fläche durch eine continuirliche Bewegung zu erzengen. Diese Umstände veranlafsten die Fürstl. Jablonowskische Gesellschaft der Wissenschaften zu Leipzig, eine neue Untersuchung der Fläche zum Gegenstande einer Preisaufgabe für das Jahr 1831 zu machen. Der Preis ist einer von mir im November 1830 eingereichten Abhandlung zuerkannt worden, die in den Actis Saciet. Jablonovianae Voi. IV. Fasc. II. pag. $204-280$. (De proprietatibus superficiei, quae hac continetur aequatione:

$$
\left(1+q^{2}\right) r-2 p q s+\left(1+p^{2}\right) t=0
$$

disquisitiones analyticae) enthalten ist, und von welcher ich jetzt einige Resultate anführen mufs, da ich mich in der gegenwärtigen Abhandlung häufiger auf dieselbe zu beziehen gezwungen bin.

Sind nämlich $x, y$, z die veränderlichen Coordinaten der Fläche und man setzt die partiellen Differentialquotienten, wie gewöhnlich:

$$
\begin{gathered}
\left(\frac{d z}{d x}\right)=p, \quad\left(\frac{d z}{d x}\right)_{y}=q ; \\
\left(\frac{d^{2} z}{d x^{2}}\right)=r, \quad\left(\frac{d^{2} z}{d x \cdot d y}\right)=s, \quad\left(\frac{d^{2} z}{d y^{2}}\right)=t ;
\end{gathered}
$$

so findet man, als Differentialgleichung der Fläche:

$$
\text { 1. } h=\left(1+q^{2}\right) r-2 p q s+\left(1+p^{2}\right) t=0 \text {. }
$$

Dem Integrale derselben hat Legendre die Form

$$
\text { 2. }\left\{\begin{array}{l}
x=\varphi^{\prime} a+\psi \prime b, \\
y=\varphi a-a \varphi^{\prime} a+\psi b-b \psi^{\prime} b, \\
z=\int v\left(-1-a^{2}\right) \varphi^{\prime \prime} a d a-\int r\left(-1-b^{2}\right) \psi^{\prime \prime} b d b,
\end{array}\right.
$$


oder, da die Integrale aus dieser Gleichung ganz entfernt werden können, die Form

$$
\text { 3. } \quad\left\{\begin{array}{l}
x=A^{3} \varphi^{\prime \prime} a-3 A a \varphi^{\prime} a+B^{3} \psi^{\prime \prime} b-3 B b \psi^{\prime} b, \\
y=-A^{3} a \varphi^{\prime \prime} a+\left(2 a^{2}-1\right) A \varphi^{\prime} a-B^{3} b \psi^{\prime \prime} b+\left(2 b^{2}-1\right) B \psi^{\prime} b, \\
z=A^{4} \varphi^{\prime \prime} a-2 A^{2} a \varphi^{\prime} a+\varphi a-B^{4} \psi^{\prime \prime} b+2 B^{2} b \psi^{\prime} b-\psi b,
\end{array}\right.
$$

gegeben, wo $a, b$ zwei beliebige Grölsen vorstellen, die aus dem Systeme der Gleichungeu (2.) oder (3.) eliminirt werden müssen; wo ferner durch $\varphi a, \psi b ; \varphi^{\prime} a, \psi^{\prime} b ; \varphi^{\prime \prime} a, \psi^{\prime \prime} b$ zwei willkürliche Functionen, resp. von $a$ und von $b$, nebst ihren beiden ersten Differentialquotienten bezeichnet sind, und Kürze halber $\sqrt{ }\left(-1-a^{2}\right)=A, r\left(-1-b^{2}\right)=B$ gesetzt ist. Durch leichte Substitutionen erhält man aus den Gleichungen (2.) die Form, deren sich Monge bedient hat:

$$
\text { 4. } \quad\left\{\begin{array}{l}
x=a+b, \\
y=\varphi a+\psi b, \\
\left.z=\int v\left(-1-\left(\varphi^{\prime} a\right)^{2}\right) d a+\int \sqrt{ }\left(-1-\left(\psi^{\prime} b\right)\right) d b\right) .
\end{array}\right.
$$

Da es mir nun eben so wenig, als den frühern Bearbeitern dieser Aufgabe, gelungen war, eine allgemeine Construction der Fläche anzugeben, so ging ich darauf aus, möglichst viele particuläre Fälle des Integrals, durch eine sich überall gleich bleibende Methode, aus der Differentialgleichung (1.) zu entdecken. Um dahin zu gelangen, wurde sie auf verschiedene Weise in zwei Gleichungen von der Art zerfällt, dafs die eine von ihnen eine leichte Integration gestattete, und die willkürlichen Functionen, die in dem Integrale derselben vorkamen, wurden dann so bestimmt, dals auch der andern Gleichung Genüge geleistet wurde. Auf diese Weise ergab sich, wenn

$$
r \text { oder } t \text { oder } r+t=0
$$

gesetzt wurde, die Gleichung der Schraubenfläche:

$$
\text { 5. } Z=y \operatorname{tang} D x \text {, }
$$

wo $D$ eine Constante bezeichnet; ferner wenn man

annahm, die Fläche, deren Gleichung

$$
s=0
$$

$$
\text { 6. } e^{D z}=\frac{\cos D y}{\cos D x}
$$

ist, aus welcher dann die allgemeinere

$$
\text { 7. } e^{B \sin (\beta-\alpha) z}=A \cdot \frac{\cos B(x \cos \alpha+y \sin \alpha+\alpha)}{\cos B(x \cos \beta+y \sin \beta+b)}
$$


188 10. Scherk, Bemerkungen über die kleinste Fläche innerhalb gegebener Grenzen.

hergeleitet wurde, wo $A, B, a, b, \alpha, \beta$ gleichfalls beliebige Constanten darstellen; ferner wenn

$$
\bar{x}=\rho \cos \vartheta, \quad y=\rho \sin \vartheta \text { und }\left(\frac{d z}{d \vartheta}\right)=0
$$

gesetzt wurde, die Umdrehungsfläche der Kettenlinie, deren Gleichung

$$
\text { 8. } \quad r\left(x^{2}+y^{2}\right)=\frac{x}{2} a\left(e^{\frac{z-b}{a}}+e^{-\frac{z-b}{a}}\right)
$$

ist, und welche Meusnier nicht durch diese Methode, sondern durch die Forderung, dafs die entstehende Fläche eine Umdrehungsfläche sein solle, erhalten hatte, und wenn man endlich

$$
\left(\frac{d^{2} z}{d Q \cdot d \vartheta}\right)=0
$$

annahm, eine Fläche, deren Gleichung

9. $Z=b \log \frac{V\left(\varrho^{2}+a^{2}\right)+V}{a} \underline{\left(\varrho^{2}-b^{2}\right)}-a \operatorname{arc}\left[\operatorname{tang}=\frac{b}{a} V\left(\frac{\varrho^{2}+a^{2}}{\varrho^{2}-b^{2}}\right)\right]+a \vartheta+c$ ist, und von welcher die Schraubenfläche und die Umdrehungsfläche der Kettenlinie specielle Fälle sind. Auch wurden für jede dieser Flächen die willkürlichen Functionen $\varphi a, \psi b$ in dem Integrale (4.) bestimmt, und im Allgemeinen gezeigt, wie man jedesmal diese willkürlichen Functionen durch Integration einer gewöhnlichen Differenzialgleichung vom ersten Grade, für welche die Bedingungen der Integrabilität erfüllt werden, bestimmen könne, wenn man auf irgend eine Weise, also z. B. durch die angewandte Zerfillungsmethode, eine endliche Gleichung erhalten hat, welche der Differentialgleichung $h=0$ Genüge leistet.

Hieraus ersieht man, dafs bis jetzt das In te gra l unserer Gleichung zur Erfindung neuer Eigenschaften, oder neuer specieller Fälle, noch nicht benutzt worden ist. Als Grund hiervon kann man annehmen, dafs es zwar leicht ist, passende Functionen $\varphi a, \varphi b$, aufzufinden, welche in den Systemen (2.) oder (4.) eine Integration gestatten: dafs es aber bei weitem schwieriger und grölstentheils unausführbar ist, diese Functionen so zu wählen, dafs man die Quantitïten $a$ und $b$ aus einem dieser Systeme eliminiren könne, und dafs das Resultat auch wirklich eine Flïche darstelle, und nicht etwa blofs eine Gleichung zwischen drei veränderlichen Grǘsen sei. Da es mir jedoch, wie erwähnt, in der angeführten Abhandlung gelungen war, die willkürlichen 'Functionen zu den bekannten Gleichungen der Flächen (5.), (6.), (8.), (9.) zu finden, so mufste sich der Gedanke darbieten, die Untersuchung umzukehren, und, 
von dem Integrale der Gleichung (1.) ausgehend, willkürliche Functionen $\varphi a, \psi b$ aufzusuchen, welche eine Elimination von $a$ und $b$ gestatten, und zu Gleichungen von neuen Flächen führen. Dies ist der Zweck, den ich mir im ersten Abschnitte dieser Untersuchung zu erreichen vorgeset: ibe. Es haben sich auf diesem Wege drei neue Flächen ergeben, deren eine überaus einfach und die Correspondirende der Fläche (6.) ist, indem sie zu jener in einer ähnlichen Beziehung steht, wie das Ellipsoid zu den Hyperboloiden. Der zweite Abschnitt dieser Abhandlung verfolgt zwar einen ganz andern Endzweck, hat jedoch mit dem ersten das gemein, dafs die Untersuchung sich gleichfalls auf dem Int egrale der Gleichung (1.) stützt. Die bisher angestelltc a Untersuchungen hatten nämlich, auf den verschiedensten Wegen, z. B. durch die bereits angeführte Zerfällung der Gleichung $(1)$ in die beiden

$$
\begin{gathered}
r+t=0, \\
\text { 10: } \quad q^{2} r-2 p q s+p^{2} t=0,
\end{gathered}
$$

oder indem $\operatorname{man} x=\rho \cos \vartheta, y=\rho \sin \delta$, und $\left(\frac{d z}{d \varrho}\right)=0$ setzte, zu dem Resultate geführt, dafs die Schraubenfläche unter den kleinsten Flächen enthalten sei. Dabei war man also ent we dè von der Entstehungsart jener Fläche durch Bewegung einer immer horizontalen geraden Linie, deren allgemeine Gleichung bekanntlich (10.) ist, wenn man die feste Ebene, der die bewegte Linie immer parallel bleiben soll, zur Coordinatenebene der $x y$ annimmt (vergl. Monge, Applic. de l'analyse à la Géom. ed. 1807. pag. 64.), oder von der Gleichung $h=0$ ausgegangen; und als einen speciellen Fall ihres Integrals hatte man $z=y \operatorname{tang} D x$ gefunden. Es fragte sich nun, ob aufser der genannten Fläche noch andere kleinste Flächen durch Bewegung einer geraden Linie entstehen könnten, müchte übrigens das Gesetz der Bewegung sein, welehes es wollte, oder mindestens, wenn etwa der Elimination Schwierigkeiten in den Weg träten, ob sich für diesen Fall nicht die Form der willkürlichen Functionen vollständig bestimmen liefse. Bei dem ersten Anblicke scheint es zwar, als bedürfe es dieser- Untersuchung gar nicht, und als könne man geradesweges behaupten, nur die Schraubenfläche könne durch Bewegung einer geraden Linie entstehen, und zugleich der Eigenschaft des Minimums der Area theilhaftig werden. Denn es seien zwei nicht in einer Ebene beflndliche gerade Linien gegeben; nimmt man ibre kleinste Entfernung 
als Axe eines geraden Cylinders an, um dessen Oberfläche eine Schraubenlinie sich hinaufwindet, und lâst durch dieselbe, und durch die Axe, eine die letztere stets unter rechtem Winkel schneidende gerade Linie sich hinaufbewegen, so ist das von der Schraubenlinie und den beiden gegebenen geraden Linien begrenzte Stück der Schraubenfläche, nach dem Vorigen, die kleinste, von den beiden gegebenen Linien begrenzte, Fläche. Liefse sich also durch dieselben noch eine andere, von der Schraubenfläche verschiedene, kleinste Fläche legen, so würde jene nicht die kleinste sein. Aber, abgesehen davon, dafs die Unmöglichkeit, dafs beide Flächen auch wohl gleichen Inhalt haben könnten, nicht von vorn herein einleuchtet: so ist offenbar Lei der angegebenen Entstehungsart auch noch die Annahme gemacht, dafs nicht blofs die durch die gegebenen beiden Geraden begrenzte Fläche, sondern auch jedes Stück derselben, welches durch eine von ihnen und durch eine andere Gerade abgeschnitten wird, die der Ebene parallel ist, welche auf der kleinsten Entfernung der beiden gegebenen Geraden senkrecht steht, oder jedes Stück, welches durch zwei solche gerade Linien begrenzt wird, ein Kleinstes sein soll. $\mathrm{Ob}$ aber diese Forderung in der Natur der Sache liege, oder eine der gegenwärtigen Frage ganze fremde Bedingung in die Rechnung bringe, scheint mir nicht leichter, als auf dem angegebenen Wege untersucht werden zu können. Hat sich nun auch das verlangte geometrische Resultat, wegen der Unmöglichkeit der Elimination, nicht ergeben, so scheint mir doch das analytische Resultat, durch welches die Form der willkürlichen Functionen in diesem Falle vollständig bestimmt wird, nicht ganz unbemerkenswerth, da man aus demselben, in Verbindung mit einem andern, später anzuführenden Umstande, mit grofser Wahrscheinlichkeit schliefsen kann, dafs es allerdings keine andere Fläche als die Schraubenfläche gebe, die, in unserm Falle, durch die Bewegung einer geraden Linie entstehen kann.

\section{2.}

Indem wir nun zur Untersuchung der Functionen, welche eine leichte Elimination gestatten, selbst, schreiten, wird man vor Allem daran denken können, den Functionen $\varphi a, \psi b$, in den Gleichungen (3.), algeb raische Werthe beizulegen, da man alsdann, nach vollbrachter Elimination, eine algebraische Gleichung für die verlangte Fläche erhielte. Aber selbst die einfachsten Annahmen führen auf endlose Weitläufigkeiten. Setzt 
man nemlich die eine derselben $\psi 6$ gleich einer Constante, so nehmen die Gleichungen (3.) die Form an :

$$
\begin{aligned}
& x=+A^{3} \varphi^{\prime \prime} a-3 A a \varphi^{\prime} a, \\
& y=-A^{3} \alpha \varphi^{\prime \prime} a+\left(2 a^{2}-1\right) A \varphi^{\prime} a, \\
& z=+A^{4} \varphi^{\prime \prime} a-2 A^{2} a \varphi^{\prime} a+\varphi a-C,
\end{aligned}
$$

aus welchen die einzige Quantität $a$ zu eliminiren ist. Man gelangt also durch diese Annahme nicht zu einer Fläche, sondern zu einer Linie. Hiernach werden

$$
\varphi a=a, \quad \psi b=b
$$

zuversichtlich die einfachsten Werthe sein, die man den willkürlichen Functionen beilegen kann; durch dieselben erhält man:

$$
\text { 11. }\left\{\begin{array}{l}
x=-3 A a-3 B b, \\
y=\left(2 a^{2}-1\right) A+\left(2 b^{2}-1\right) B, \\
z=-2 A^{2} a+a+2 B^{2} b-b,
\end{array}\right.
$$

oder, nach Elimination von $A=r\left(-1-a^{2}\right)$ und $B=r\left(-1-b^{2}\right)$,

$$
\begin{aligned}
& \left(\frac{\left(2 a^{2}-1\right) x+3 a y}{(1+2 a b)(a-b)}\right)^{2}+9\left(1+b^{2}\right)=0, \\
& \left(\frac{\left(2 b^{2}-1\right) x+3 b y}{(1+2 a b)(a-b)}\right)^{2}+9\left(1+a^{2}\right)=0, \\
& z-(a-b)\left(3+2 a^{2}+2 a b+2 b^{2}\right)=0 .
\end{aligned}
$$

Aber der Versuch, $a$ und $b$ aus diesen Gleichungen zu eliminiren, ist fast unausführbar, und um so weniger anzustellen, als das Resultat, gesetzt auch, es stellte wirklich eine Fläche dar, doch von so hohem Grade sein würde, dals man an eine Benutzung dieser Fläche zu dem Zwecke, um dessentwillen die speciellen Flächen allein gesucht werden, nicht im Entferntesten denken könnte; der Zweck kann offenbar kein anderer sein, als: durch die Zusammenstellung der gemeinschaftlichen Eigenschaften verschiedener specieller Flächen, zu einer Eigenschaft der allgemeinen Fläche zu gelangen. Noch ungleich weitläufiger wird die Rechnung, wenn man den willkürlichen Functionen Werthe beilegt, deren zweite Differentialquotienten nicht verschwinden; und so wird sich aus den Gleichungen (3.), trotz dem, dafs die Integrale aus ihnen verschwunden sind, wohl kaum ein bemerkenswerthes Resultat herleiten lassen.

\section{3.}

Wenden wir uns nun zu den Gleichungen (2.), welche vor der Form (4.) den Vorzug haben, dafs in ihnen die irrationale Quantität $\checkmark\left(1+a^{2}\right)$ verkömmt, welche offenbar für die Rechnung bequemer ist, als 
192 10. Scherk, Bemerkungen über die kleinste Fläche innerhalb gegebener Grenzen. die: $r\left[1+\left(\varphi^{\prime} a^{2}\right)\right]$. Setzt man $\varphi^{\prime} a=i A$, wo $A$ eine willkürliche Function von $a$ bezeichnet, und $i^{2}=-1$ ist, so wird $\varphi a-a \varphi^{\prime} a=-\int a \varphi^{\prime \prime} a \cdot d a$ $=-i \int a d A=-i \int a A^{\prime} d a$ und $i \int v\left(1+a^{2}\right) \varphi^{\prime \prime} a d a=-\int v\left(1+a^{2}\right) A^{\prime} d a$; macht man die ähnlichen Annahmen in Beziehnng auf $b$, so wird

$$
\text { 12. }\left\{\begin{array}{l}
x=i A+i B, \\
y=-i \int a A^{\prime} d a-i \int b B^{\prime} d b, \\
z=-i \int v\left(1+a^{2}\right) A^{\prime} d a+\int v\left(1+b^{2}\right) B^{\prime} d b .
\end{array}\right.
$$

Unter dieser Form wollen wir hier beständig das Integral der Gleichuug (1.) gebrauchen. Die zuerst sich darbietende Annahme ist auch hier offenbar: $A^{\prime}$ und $B^{\prime}$ Constanten gleich zu setzen. Es sei also

hierdurch erhält man:

$$
A^{\prime}=C, \quad B^{\prime}=C^{\prime} \text {; }
$$

$$
\begin{aligned}
& x=C i a+C^{\prime} i b, \\
& y=-\frac{C i a^{2}}{2}-\frac{C^{\prime} i b^{2}}{2}, \\
& z=-\frac{C a V\left(1+a^{2}\right)}{2}+\frac{C^{\prime} b V\left(1+b^{2}\right)}{2}+\frac{x}{2} C \log \left[V\left(1+a^{2}\right)-a\right] \\
& -\frac{1}{2} C^{\prime} \log \left[V\left(1+b^{2}\right)-b\right] .
\end{aligned}
$$

Setzt man hier, um den einfachsten Fall zu exhalten, $C^{\prime}=-C$, und $x$, $y$, z resp. für $\frac{x}{C}, \frac{y}{C}, \frac{z}{z}$, so wird

$$
\begin{aligned}
& x=i(a-b), \\
& y=-\frac{i}{2}\left(a^{2}-b^{2}\right), \\
& z-\frac{1}{2} a r\left(1+a^{2}\right)+\frac{1}{2} b r\left(1+b^{2}\right)=\frac{x}{2} \log \left\{\left[r\left(1+a^{2}\right)-a\right]\left[r\left(1+b^{2}\right)-b\right]\right\},
\end{aligned}
$$

folglich

13. $\frac{1}{2} e^{2 z+a V\left(1+a^{2}\right)+b V\left(1+b^{2}\right)}+\frac{\pi}{2} e^{-2 z-a V\left(1+a^{2}\right)-b V\left(1+b^{2}\right)}=\sqrt{ }\left(1+a^{2}\right) \sqrt{ }\left(1+b^{2}\right)+a b$. Nun aber ist

$$
a=-\frac{y}{x}-\frac{i x}{2}, \quad b=-\frac{y}{x}+\frac{i x}{2},
$$

also

$$
1+a^{2}=t+i y, \quad 1+b^{2}=t-i y,
$$

wenn, Kürze halber,

$$
\text { 14. } 1+\frac{y^{2}}{x^{2}}-\frac{x^{2}}{4}=t
$$

gesetzt wird. Setzt man nun

folglich

$$
t=\rho \cos \vartheta, \quad y=\rho \sin \vartheta
$$


10. Scherk, Bemerkungen über die kleinste Fläche innerhalb gegebener Grenzen.

so wird

$$
\text { 15. } \rho^{2}=t^{2}+y^{2},
$$

$$
r\left(1+a^{2}\right)=\rho^{\frac{\pi}{3}}\left(\cos \frac{x}{2} \vartheta+i \sin \frac{x}{2} \vartheta\right), \quad r\left(1+b^{2}=\rho^{\frac{\pi}{2}}\left(\cos \frac{x}{2} \vartheta-i \sin \frac{x}{2} \vartheta\right),\right.
$$

also

$$
\begin{aligned}
a r\left(1+a^{2}\right)+b r\left(1+b^{2}\right) & =-\frac{2 \varrho^{\frac{2}{2}} y}{x} \cos \frac{1}{2} \vartheta+2 \rho^{\frac{1}{2}} x \sin \frac{1}{2} \vartheta \\
& =-\frac{y}{x} r(2 \rho+2 t)+x r(2 \rho-2 t), \\
r\left(1+a^{2}\right) r\left(1+b^{2}\right)+a b & =\rho+\frac{y^{2}}{x^{2}}+\frac{x^{2}}{4} .
\end{aligned}
$$

Werden also diese Resultate in (13.) gesetzt, so erhält man:

16. $\frac{x}{2} e^{2 z-\frac{y}{x} ! \sqrt[V]{ }(2 \rho+2 t)+x \sqrt[V]{ }(2 \rho-2 t)}+\frac{x}{2} e^{-2 x+\frac{y}{x} \sqrt[V]{ }(2 \rho+2 t)-x \sqrt[V]{ }(2 \rho-2 t)}=\rho+\frac{y^{2}}{x^{2}}+\frac{x^{2}}{4}$, in wecher Gleichung $t$ und $\rho$ durch (14.) und (15.) bestimmt, und $\frac{x}{C}$, $\frac{y}{C}, \frac{z}{C}$ resp. für $x, y, z$ gesetzt werden müssen. Dies ist also dex Gleichung einer neuen, von den bisher bekannten sich sehr wesentlich unterscheidenden, Fläche.

Man versuche hierauf, für die willkürlichen Functionen ganze Potenzen zu setzen. Man kann aber gleich Anfangs bemerken, dafs man dann von den negativen ganzen Potenzen nur die $(-1)^{\text {te }}$ zu untersuchen braucht. Ist nämlich erstens

$$
A^{\prime}=C a^{m}, \quad B^{\prime}=C^{\prime} b^{n},
$$

wo $C$ und $C^{\prime}$ beliebige Constanten bezeichnen, so erhält man

$$
\begin{aligned}
& x=\frac{C i a^{m+1}}{m+1}+\frac{C^{\prime} i b^{n+1}}{n+1}, \\
& y=-\frac{C i a^{m+2}}{m+2}-\frac{C^{\prime} i b^{n+2}}{n+2}, \\
& z=-C \int r\left(1+a^{2}\right) \cdot a^{m} d a+C^{\prime} \int r\left(1+b^{2}\right) \cdot b^{n} d b .
\end{aligned}
$$

Ist hingegen zweitens

so wird

$$
A^{\prime}=\frac{C}{a^{m+3}}, \quad B^{\prime}=\frac{C^{\prime}}{b^{n+3}},
$$

$$
\begin{aligned}
& x=-\frac{C_{i}}{(m+2) a^{m+2}}-\frac{C^{\prime} i}{(n+2) b^{n+2}}, \\
& y=\frac{C i}{(m+1) a^{m+1}}+\frac{C^{\prime} i}{(n+1) b^{n+1}} \\
& z=-C \int \frac{V\left(1+a^{2}\right)}{a^{m+3}} d a+C^{\prime} \int \frac{V\left(1+b^{2}\right)}{b^{n+3}} d b
\end{aligned}
$$

Crelle's Journal d. M. Bd. XIII. Hft. 3. 
194 10. Scherk, Bemerkungen über die kleinste Fläche innerhalb 'gegebener Grenzen.

Wird aber in diesen Glcichungen $\frac{1}{a}, \frac{1}{b}$ resp. an die Stelle von $a, b$ gesetzt, so erhält man ganzi dieselben Werthe für $y, x,-z$, die man bei der ersten Annahme für $x, y, z$ erhalten hatte. Die Resultate werden sich also auch nur auf die so eben angegebene Weise von einander unterscheiden, und folglich dieselben reellen oder imaginairen Flächen darstellen. Setzt man nun

so wird

$$
m=-1,0,1,2,3, \ldots
$$

$$
-(m+3)=-2,-3,-4,-5,-6, \ldots
$$

woraus hervorgeht, dafs, mit Ausnahme der $(-1)^{\text {ten }}$ und $(-2)^{\text {ten }}$, die eben dasselbe Resultat geben, jede negative Potenz durch eine positive repräsentirt wird.

Es sei also gegenwärtig

$$
A^{\prime}=\frac{c}{a}, \quad B^{\prime}=\frac{C^{\prime}}{b},
$$

so erhält man auch hier den bei weiten einfachsten Fall, wenn man $C^{\prime}=-C$ setzt. Hierdurch wird

$$
\begin{aligned}
& x=i \log \frac{a}{b}, \\
& y=-i(a-b), \\
& z+r\left(1+a^{2}\right)+r\left(1+b^{2}\right)=\log \left\{\left[V\left(1+\frac{1}{a^{2}}\right)+\frac{1}{a}\right]\left[V\left(1+\frac{1}{b^{2}}\right)+\frac{1}{b}\right]\right\},
\end{aligned}
$$

wo Kürze halber $x, y, z$ für $\frac{x}{C}, \frac{y}{C}, \frac{z}{c}$ gesetzt sind.' Die letzte Gleichung giebt :

17. $\frac{x}{2} e^{z+V\left(1+a^{2}\right)+V\left(1+b^{2}\right)}+\frac{7}{2} e^{-z-V\left(1+a^{2}\right)-\left(1+b^{2}\right)}=\frac{V\left(1+a^{2}\right) V\left(1+b^{2}\right)+1}{a b}$.

Da aber $e^{-i x}=\frac{a}{b}$, also $b=a e^{i x}$, so ist

daher

$$
i y=a-b=-2 i a e^{\frac{i x}{2}} \sin \frac{x}{2} x
$$

und

$$
a=-\frac{y e^{-\frac{1}{i} i x}}{2 \sin \frac{x}{2} x}, \quad b=-\frac{y e^{\frac{\pi}{2} i x}}{2 \sin \frac{x}{2} x}
$$

wenn

$$
1+a^{2}=\frac{t-i y^{2} \sin x}{4 \sin \frac{1}{2} x^{2}}, \quad 1+b^{2}=-\frac{t+i y^{2} \sin x}{4 \sin \frac{x}{2} x^{2}},
$$

gesetzt wird. Nun sei

$$
\text { 18. } 4 \sin \frac{\pi}{2} x^{2}+y^{2} \cos x=t
$$

$$
t=\rho \cos \vartheta, \quad y^{2} \sin x=\rho \sin \vartheta
$$


also

so wird

$$
\text { 19. } \rho^{2}=t^{2}+y^{4} \sin x^{2} \text {, }
$$

und

$$
\sqrt{ }\left(1+a^{2}\right)+V\left(1+b^{2}\right)=\frac{\rho^{\frac{1}{2}} \cos \frac{1}{2} \vartheta}{\sin \frac{x}{2} x}=\frac{V\left(\frac{x}{2} \varrho+\frac{1}{2} t\right)}{\sin \frac{x}{2} x}
$$

$$
r\left(1+a^{2}\right) r\left(1+b^{2}\right)=\frac{\varrho}{4 \sin \frac{1}{2} x^{2}}
$$

Werden also diese Werthe in (17.) substituirt, so erhält man

$$
\text { 20. } \frac{1}{2} e^{z+\operatorname{cosec} \frac{1}{2} x V\left(\frac{1}{2} \rho+\frac{1}{2} t\right)}+\frac{1}{2} e^{-z-\operatorname{cosec} \frac{1}{2} x V\left(\frac{1}{2} \rho+\frac{1}{t} t\right)}=\frac{4 \sin \frac{1}{2} x^{2}+\varrho}{y^{2}} \text {, }
$$

wo $t$ und $\rho$ durch (18.) und (19.) bestimmt werden, und $\frac{x}{c}, \frac{y}{C}, \frac{z}{c}$ für $x, y, z$ zu setzen ist. Dies ist also abermals eine neue, der Gleichung $h=0$ Genüge leistende, Fläche. .

Man setze drittens

$$
5 .
$$

$$
A^{\prime}=C a, \quad B^{\prime}=C^{\prime} b
$$

so wird

$$
\begin{aligned}
& x=+\frac{1}{2} i\left(C a^{2}+C^{\prime} b^{2}\right) \\
& y=-\frac{1}{3} i\left(C a^{3}+C^{\prime} b^{3}\right) \\
& z=-\frac{1}{3} C\left(1+a^{2}\right)^{\frac{3}{2}}+\frac{1}{3} C^{\prime}(1+b)^{\frac{3}{2}}
\end{aligned}
$$

und das Resultat der Elimination von $a$ und $b$ ist also gegenwärtig, wie in allen den Fällen, wo $A^{\prime}=C a^{2 n-1}$ oder $=\frac{C}{a^{2 n+2}}$ gesetzt, und eine ähnliche Annahme für $B^{\prime}$ gemacht wird, in einer algebraischen Gleichung enthalten. Aber selbst in dem einfachsten Falle, der auch hier wieder in $C+C^{\prime}=0$ enthalten ist, führt die Elimination von $b$ auf 2 Gleichungen für $a$ vom vierten und vom sechsten Grade, so, dafs es nicht die Mühe lohnt, die Rechnung weiter fortzusetzen. Mit nooh geringerem Erfolge wird man es versuchen, $A^{\prime}=C a^{2}, C a^{3}$ u. s. fo anzunehmen.

6.

Es sei viertens $A^{\prime}=\frac{C}{1+a^{2}}$, so wird

$$
\begin{aligned}
A & =C \operatorname{arc}(\operatorname{tang}=a), \\
\int a A^{\prime} d a & =\frac{1}{2} C \log \left(1+a^{2}\right), \\
\int \sqrt{ }\left(1+a^{2}\right) A^{\prime} d a & =C \log \left[V\left(1+a^{2}\right)-a\right] .
\end{aligned}
$$

Macht man nun die ähnliche Annahme für $B^{\prime}$, und setzt Kürze halber $\operatorname{arc}(\operatorname{tang}=a)=\alpha, \operatorname{arc}(\operatorname{tang} \equiv b)=\beta$, so wird 
196 10. Scherk, Bemerkungen über die.kleinste Fläche innerhalb gegebener Grenzen.

$$
\begin{aligned}
& x=i\left(C \alpha_{1}^{\prime}+C^{\prime} \beta\right), \\
& y=i\left(C \log \cos \alpha+C^{\prime} \log \cos \beta\right), \\
& z=C \log \operatorname{tang}\left(\frac{\pi}{4} \pi-\frac{1}{2} \alpha\right)+C^{\prime} \log \operatorname{tang}\left(\frac{r}{4} \pi+\frac{r}{2} \beta\right) .
\end{aligned}
$$

Hier ist, wie man leicht bemerken wird, nur in den Fällen an eine Elimination zu denken, wo entweder $C=C^{\prime}$, oder $C=-C^{\prime}$.

Es sei also erstens $C=C^{\prime}=\frac{1}{2 D_{i}} ;$ auch setze man $2 D a$ und $2 D b$ resp. für $\alpha, \beta$, so wird

$$
\text { 21. }\left\{\begin{array}{l}
x=a+b, \\
y=\frac{1}{2 D} \log (\cos 2 D \cdot a \cdot \cos 2 D b), \\
z=\frac{-i}{2 D} \log \left[\operatorname{tang}\left(\frac{x}{4} \pi-D a\right) \cdot \operatorname{tang}\left(\frac{x}{4} \pi+D b\right)\right],
\end{array}\right.
$$

aus welchen Gleichungen nun $a$ und $b$ zu eliminiren ist. Aber dies sind ganz dieselben Gleichungen, die in meiner oben angeführten Abhandlung pag. 274 angegeben sind, nur dafs $y$ mit $z$ verwechselt ist. Wie dort, folgt auch aus denselben:

$$
e^{D y}=\frac{\cos D x}{\cos D z}
$$

welches die bereits bekannte Fläche (6.) ist, da es erlaubt ist, die Coordinaten mit einander zu verwechseln.

Es sei zweitens $C=-C^{\prime}=\frac{1}{2 D i}$, und es werde abermals $\alpha$ und $\beta$ resp. für $a$ und $b$ gesetzt, so wird

$$
\text { 22. }\left\{\begin{array}{l}
x=a-b, \\
y=\frac{1}{2 D} \log \frac{\cos 2 D a}{\cos 2 D b}, \\
z=\frac{1}{2 D i} \log \left[\operatorname{tang}\left(\frac{\pi}{4} \pi-D a\right) \operatorname{tang}\left(\frac{\pi}{4} \pi-D b\right)\right] \text {. }
\end{array}\right.
$$

Um aus dieser Gleichung $a$ und $b$ zu eliminiren, wollen wir zuerst einen ganz directen Weg einschlagen. Es ist

$$
e^{2 D y}=\frac{1-\operatorname{tang} D a^{2}}{1+\operatorname{tang} D a^{2}} \cdot \frac{1+\operatorname{tang}}{1-\operatorname{tang}} \frac{D b^{2}}{D b^{2}}=\frac{1-\alpha^{2}}{1+a^{2}} \cdot \frac{1+\operatorname{tang}(D a-D x)^{2}}{1-\operatorname{tang}(D a-D x)^{2}},
$$

wenn Kürze halber $\operatorname{tang} D a=\alpha$ gesetzt wird. Setzt man auf gleiche Weise $\operatorname{tang} D x=\xi$, so hat man also

$$
\frac{1+\operatorname{tang}(D a-D x)^{2}}{1-\operatorname{tang}(D a-D x)^{2}}=\frac{(1+\alpha \zeta)^{2}+(\alpha-\zeta)^{2}}{(1+\alpha \zeta)^{2}-(\alpha-\zeta)^{2}}=\frac{\left(1+\alpha^{2}\right)\left(1+\zeta^{2}\right)}{\left(1-a^{2}\right)\left(1-\zeta^{2}\right)+4 \alpha \zeta}
$$

$$
e^{2 D y}=\frac{\left(1-\alpha^{2}\right)\left(1+\zeta^{2}\right)}{\left(1-\alpha^{2}\right)\left(1-\zeta^{2}\right)+4 \alpha \zeta}=\frac{1-\alpha^{2}}{\left(1-\alpha^{2}\right) \cos 2 D x+2 \alpha \sin 2 D x},
$$


oder

$$
\text { 23. } e^{-2 D y}=\cos 2 D x-\frac{2 \sin 2 D x}{\alpha-\alpha^{-1}} \text {. }
$$

Ferner geben die Gleichungen (22.)

$$
e^{2 D z . i}=\operatorname{tang}\left(\frac{1}{4} \pi-D a\right) \operatorname{tang}\left(\frac{1}{4} \pi-D a+D x\right)=\frac{1-\alpha}{1+\alpha} \cdot \frac{1+\zeta+\alpha+\alpha \zeta}{1-\zeta+\alpha+\alpha \zeta}
$$

woraus sich leicht, wenn

gesetzt wird,

$$
\text { 24. } \rho^{2}=\zeta^{2}+\sec D z^{2}
$$

ergiebt. Hieraus folgt

$$
\alpha=\frac{\varrho-1}{\zeta+i \operatorname{tang} D z}
$$

$$
\frac{1}{2}\left(\alpha-\frac{1}{\alpha}\right)=\frac{(\varrho-1)^{2}-(\zeta+i \operatorname{tang} D z)^{2}}{2(\varrho-1)(\zeta+i \operatorname{tang} D z)}=\frac{\sec D z-\rho \cos D z-\zeta \sin D z . i}{(\varrho-1)(\zeta \cos D z+\sin D z . i)} .
$$

Multiplicirt man dieses Ausdrucks Zähler und Nenner durch

$$
\sec D z-\rho \cos D z+\zeta \sin D z . i \text {, }
$$

so wird, nach leichten Umformungen, der Zähler $=(\rho-1)^{2}$, und, nach Weglassung des Factors $\rho-1$, wird der Nenner

$$
=-(\varrho-1) \zeta \cos D z^{2}+\sin D z \cos D z\left[\xi^{2}+\sec D z^{2}-\rho\right] i \text {, }
$$

also, in Folge von (24.),

$$
=(\rho-1)\left[-\zeta \cos D z^{2}+\rho \sin D z \cos D z . i\right]
$$

folglich haben Zähler und Nenner von $\frac{r}{2}\left(\alpha-\frac{1}{\alpha}\right)$ den gemeinschaftlichen Factor $(\rho-1)^{2}$, und man erhält

$$
\frac{2}{a-\alpha^{-1}}=-\zeta \cos D z^{2}+\rho \sin D z \cos D z . i \text {. }
$$

Setzt man nunmehr diesen Werth in (23.), und restituirt für $\zeta$ seinen Werth $\operatorname{tang} D_{x}$, so ergiebt sich

$$
\text { 25. } \quad e^{-2 D y}=1-2 \sin D x^{2} \sin D z^{2}-\frac{x}{2} \varrho \sin 2 D x \sin 2 D z . i \text {, }
$$

als Resultat der Elimination von $a$ und $b$ aus den Gleichungen (22.). Hier sieht man nun sogleich, dafs, für ein reelles $D$, diese Gleichung eine Linie und nicht eine Fläche darstellt. Denn, da sie die Form $U+V i=0$ hat, so mufs $U=0, V=0$ sein; diese beiden Gleichungen stellen also eine Linie dar, ausgenommen, wenn die eine derselbęn durch die andere befriedigt wird. Dies ist aber offenbar hier unmöglich, da $U$ Exponentialgrölsen und Kreisfunctionen, $V$ aber nur Kreisfunctionen enthält.

$$
7 .
$$

Man setze aber in dieselbe Gleichung (25.) $D i$ statt $D$, so nimmt sie die Form 
198 10. Scherk, Bemerkungen über die kleinste Fläche innerhalb gegebener Grenzen.

$\cos 2 D y-i \sin 2 D y=1-2\left(\frac{e^{D x}-e^{-D x}}{2}\right)^{2}\left(\frac{e^{D z}-e^{-D z}}{2}\right)^{2}+\frac{x}{8} \varrho\left(e^{2 D x}-e^{-2 D x}\right)\left(e^{2 D z}-e^{-2 D z}\right) i$ an, wo

oder, indem man

$$
\rho^{2}=1-\left(\frac{e^{D x}-e^{-D x}}{e^{D x}+e^{-D x}}\right)^{2}-\left(\frac{e^{D z}-e^{-D z}}{e^{D z}+e^{-D z}}\right)^{2},
$$

$$
\begin{array}{ll}
\frac{e^{D x}+e^{-D x}}{2}=\gamma, & \frac{e^{D x}-e^{-D x}}{2}=\gamma^{\prime}, \\
\frac{e^{D z}+e^{-D z}}{2}=\delta, & \frac{e^{D z}-e^{-D z}}{2}=\delta^{\prime},
\end{array}
$$

setzt,

$$
\text { 26. } \quad \sin D y^{2}-y^{\prime 2} \delta^{\prime 2}+\left[\sin D y \cos D y+\rho \gamma \delta \gamma^{\prime} \delta^{\prime}\right] i=0 \text {. }
$$

Auch diese Gleichung hat also die Form $U+V i=0$, und sie würde demnach gleichfalls eine Linie darstellen, wenn nicht dadurch, dafs $U=0$ gesetzt wird, zu gleicher Zeit die Gleichung $V=0$ befriedigt würde. Dies geschieht aber in der That. Denn es sei

$$
\text { 2\%. } \sin D y^{2}=\gamma^{\prime 2} \delta^{\prime 2} \text {, }
$$

so wird hierdurch der Factor von $i$, in (26.), =

Da aber

$$
\gamma^{\prime} \delta^{\prime}(\cos D y \pm \rho \gamma \delta) \text {. }
$$

so ist

$$
\gamma^{2}-\gamma^{\prime 2}=\delta^{2}-\delta^{\prime 2}=1 \text {, }
$$

$$
\cos D \gamma^{2}=1-\gamma^{\prime 2} \delta^{\prime 2}=\gamma^{2} \delta^{2}\left(1-\frac{\gamma^{\prime 2}}{\gamma^{2}}-\frac{\delta^{\prime 2}}{\delta^{2}}\right)=\gamma^{2} \delta^{2} \rho^{2},
$$

und folglich

$$
\cos D y \pm \rho \gamma \delta=0
$$

Es verschwindet also dadurch, dafs der reelle Theil von (26.) $=0$ gesetzt wird, auch der imaginäre, und demnach ist (27.)

$$
\text { 28. } \sin D y= \pm\left(\frac{e^{D x}-e^{-D x}}{2}\right)\left(\frac{e^{D z}-e^{-D z}}{2}\right)
$$

die Gleiahung einer neuen, der Gleichung $h=0$ Genüge leistenden, Fläche, wo es gleichgültig ist, welches Vorzeichen man dem zweiten Theile vorsetzt, da das eine in das andere übergeht, wenn man $D$ in $-D$ verwandelt.

Aus dem Bisherigen geht also hervor, dafs, da die Gleichung (27.) sowohl dem reellen, als dem imaginären Theile von (26.) besonders Genüge leistet, diese unter die Form

$$
\left(\sin D y^{2}-\gamma^{\prime 2} \delta^{\prime 2}\right)^{p}\left[\left(\sin D y^{2}-y^{\prime 2} \delta^{\prime 2}\right)^{1-p}+W^{i}\right]=0
$$

muls gesetzt werden können, wo $W$ eine noch unbekaunte Function von $x, y, z$, und $p$ einen positiven, ächten Bruch vorstellt, der auch $=1$ sein 
10. Scherk, Bemerkungen über die kleinste Fläche innerhalb gegebener Grenzen. 199

kann. Dieser Gleichung wird nun zwar auch Genüge geleistet, wenn man den zweiten Factor besonders $=0$ setzt. Da aber die hieraus sich ergebende Gleichung auf keine Weise eine Fläche darstellen kann, so ist die Kenntri is desselben um so mebr überflüssig, als sich leicht übersehen lälst, dafs er nur ein, durch die Elimination von $a$ und $b$ in die Rechnung gekommener, überflüssiger Factor ist. Man kann nämlich die Gleichung (28.) aus (22.), ohne dafs sie mit jenem Factor behaftet wäre, herleiten. Dies geht auf folgende Weise an. Setzt man in die drei Gleichungen (22.) $D i$ statt $D$, so geht die zweite in folgende über:

oder, wenn

$$
y=\frac{1}{2 D i} \log \frac{e^{2 D a}+e^{-2 D a}}{e^{2 D b}+e^{-2 D b}}
$$

$$
\begin{aligned}
e^{2 D a}+e^{-2 D a} & =F, & e^{2 D b}+e^{-2 D b} & =G, \\
e^{D a}+e^{-D a} & =f, & e^{D b}+e^{-D b} & =g^{g}, \\
e^{D a}-e^{-D a} & =f^{\prime}, & e^{D b}-e^{-D b} & =g^{\prime},
\end{aligned}
$$

gesetzt wird, so ist

also

$$
e^{D y i}=\sqrt{ }\left(\frac{F}{g}\right)
$$

$$
\text { 29. } \quad i \sin D y=\frac{\mathrm{I}}{2} V\left(\frac{F}{G}\right)-\frac{\mathrm{I}}{2} V\left(\frac{G}{F}\right)=\frac{F-G}{2 V(F g)} \text {. }
$$

Auf diese Weise wird die dritte Gleichung (22.):

$$
e^{-2 D z}=\frac{1-\operatorname{tang} D i a}{1+\operatorname{tang} D i a} \cdot \frac{1-\operatorname{tang} D i b}{1+\operatorname{tang} D i b}=\frac{f-i f^{\prime}}{f+i f^{\prime}} \cdot \frac{g-i g^{\prime}}{g+i g^{\prime}}=\frac{\left(f-i f^{\prime}\right)^{2}\left(g-i g^{\prime}\right)^{2}}{\left(f^{2}+f^{\prime 2}\right)\left(g^{2}+g^{\prime^{2}}\right)},
$$

folglioh

$$
e^{-D z}= \pm \frac{f-i f^{\prime}}{\sqrt{\left(f^{2}+f^{\prime 2}\right)}} \cdot \frac{g-i g^{\prime}}{\sqrt{\left(g^{2}+i g^{\prime 2}\right)}}, \text { und } e^{D z}= \pm \frac{f+i f^{\prime}}{\sqrt{\left(f^{2}+f^{\prime 2}\right)}} \cdot \frac{g+i g^{\prime}}{\sqrt{\left(f^{2}+f^{\prime 2}\right)}},
$$

wo beide Male dieselben Vorzeichen zu setzen sind. Demnach ist

$$
\frac{e^{D z}-e^{-D z}}{2}= \pm \frac{\left(f^{\prime} g+f g^{\prime}\right) i}{\sqrt{ }\left(f^{2}+f^{\prime 2}\right) V\left(g^{2}+g^{\prime 2}\right)}
$$

oder, wenn man für $f, g, \ldots$ ihre Werthe setzt, und bemerkt, dafs $f^{2}+f^{\prime 2}=2 F, g^{2}+g^{\prime 2}=2 G$ :

Multiplicirt man diese Gleichung mit

$$
\frac{e^{D z}-e^{-D z}}{2}= \pm \frac{e^{D(\alpha+b)}-e^{-D(a+b)}}{V(F G)} i
$$

$$
\frac{e^{D x}-e^{-D x}}{2}=\frac{e^{D(a-b)}-e^{-D(-b)}}{2}
$$

so wird das Product der zweiten Theile

$$
= \pm \frac{\left.e^{2 D t}+e^{-2 D a}-e^{2 D b}-e^{-2 D b}\right)}{2 V(F G)} i=\frac{(F-G)}{2 V(F G)} i= \pm \sin D y \text { (wegen (29.)); }
$$


200 10. Scherk, Bemerkungen über die kleinste Fläche innerhalb gegebener Grenzen.

folglich ist

mit (28.) übereinstimmend.

$$
\sin D y= \pm \frac{e^{D x}-e^{-D x}}{2} \cdot \frac{e^{D z}-e^{-D z}}{2}
$$

Verwechselt man dieZeichen der veränderlichen Coordinaten, und setzt

$$
\text { 30. } \sin D z= \pm \frac{e^{D x}-e^{-D x}}{2} \cdot \frac{e^{D y}-e^{-D y}}{2} \text {, }
$$

so findet man für die Krümmungshalbmesser dieser Fläche folgenden einfachen Ausdruck:

$$
R= \pm \frac{1}{D} \cdot \frac{e^{D x}+e^{-D x}}{2} \cdot \frac{e^{D y}+e^{-D y}}{2}= \pm \frac{1}{4 D} \cdot \frac{\sin D z^{\prime}}{\sin D z}
$$

wo $z^{\prime}$ die dritte Ordinate des Punctes anzeigt, dessen beiden andern Coordinaten $2 x$ und $2 y$ sind.

\section{8.}

Es ist bereits oben bemerkt worden, dafs man die Gleichung $c^{D y}=\frac{\cos D x}{\cos D z}$ aus (21.), also $e^{D z}=\frac{\cos D y}{\cos D x}$, aus Monge's Form (4.) erhält, wenn man

31. $\varphi a=\frac{i}{2 D} \log \operatorname{tang}\left(\frac{\mathrm{I}}{4} \pi-D a\right), \quad \psi b=-\frac{i}{2 D} \log \operatorname{tang}\left(\frac{\pi}{4} \pi-D b\right)$ setzt, und beiden Wurzelgröfsen in $z$ dasselbe Vorzeichen gieht. Ferner haben wir die Gleichung (28.) aus (22.) hergeleitet. Verwechselt man also $y$ und $z$, und setzt $-b$ statt $b$, so sieht man, dafs

$$
\sin D z= \pm \frac{e^{D x}-e^{-D x}}{2} \cdot \frac{e^{D y}-e^{-D y}}{2}
$$

das Resultat der Elimination von $a$ und $b$ aus den Gleichungen

$$
\begin{aligned}
& x=a+b, \\
& y=-\frac{1}{2 D} \log \operatorname{tang}\left(\frac{1}{4} \pi-D i a\right)+\frac{1}{2 D} \log \operatorname{tang}\left(\frac{\pi}{4} \pi-D i b\right), \\
& z=\frac{1}{2 D i} \log \cos 2 D i a-\frac{1}{2 D i} \log \cos 2 D i b
\end{aligned}
$$

ist, welche abermals die Form der Gleichungen (4.) haben, indem

32. $\varphi a=-\frac{1}{2 D} \log \operatorname{tang}\left(\frac{1}{4} \pi-D i a\right), \quad \psi b=\frac{1}{2 D} \log \operatorname{tang}\left(\frac{1}{4} \pi-D i b\right)$ gesetzt wird, und den Wurzelgrölsen in $z$ entgegengesetzte Zeichen beigelegt werden. Beide Annahmen unterscheiden sich also blofs dadurch, dals das erste Mal den Wurzelgröfsen dasselbe, das zweite Mal verschiedene Vorzeichen vorgesetzt, und in diesem Falle ein imaginärer, in jenem ein reeller Parameter angenommen werden mufste, um eine reelle Fläche zu érhalten. Diese Bemerkung führte uns darauf, zu versuchen, 
10. Scherk, Bemerkungen über die kleinste Fläche innerhalb gegebener Grenzen. 201

ob sich noch neue Flächen ergeben, wenn man, statt, wie in diesen beiden Fällen (31.) und (32.), den willkürlichen Functionen verschiedene Vorzeichen beizulegen, ihnen dasselbe giebt, dann den Wurzelgröfsen in $z$ abwechselud dasselbe oder ein rerschiedenes Zeichen vorsetzt, und den Parameter in allen Fällen sowohl reell als imaginïr anuimmt.

Diese Annahmen haben folgende Resultate gegeben: Aus den Gleichungen

$$
\begin{aligned}
x & =a+b, \\
y & =\frac{i}{2 D} \log \operatorname{tang}\left(\frac{\mathrm{r}}{4} \pi-D a\right)+\frac{i}{2 D} \log \operatorname{tang}\left(\frac{\mathrm{T}}{4} \pi-D b\right) \\
z & = \pm \frac{1}{2 D} \log (\cos 2 D a \cdot \cos 2 D b)
\end{aligned}
$$

folgt, durch Elimination von $a$ und $b$ :

$$
\text { 33. } \pm 2 \sin D z=\frac{e^{D y}-e^{-y D}}{e^{D x}-e^{-D x}}+\frac{e^{D x}-e^{-D x}}{e^{D y}-e^{-D y}} \text {. }
$$

Nimmt man zweitens $x$ und $y$ wie vorher an, setzt aber

so erhält man

$$
t= \pm \frac{1}{2 D} \log \frac{\cos 2 D a}{\cos 2 D b}
$$

$$
\text { 34. } \pm \cos D z=\frac{e^{D x}+e^{-D x}}{2} \cdot \frac{e^{D y}+e^{-D y}}{2} \text {. }
$$

Auch bemerke man noch, daís die auf einem andern Wege, als dem bisherigen, herzuleitende Gleichung

$$
\text { 35. } \pm 2 \cos D z=\frac{e^{D x}+e^{-D x}}{e^{D y}+e^{-D y}}+\frac{e^{D y}+e^{-D y}}{e^{D x}+e^{-D x}}
$$

der Gleichung (1.) Genüge leistet. Aber man übersieht sehr leicht, dafs, mau mag den Parameter $D$ reell oder imaginär annehmen, keine dieser Gleichungen (33.), (34.), (35.) eine F läich e darstellt, da für reelle Werthe von $x$ und $y, \sin D z$ und $\cos D z$ grïfser als die Einheit werden.

Aufser den bisher untersuchten habe ich noch folgende Annahmen: $A^{\prime}=\frac{C a}{1+a^{2}}, \quad \frac{C}{a\left(1+a^{2}\right)}, \quad \frac{C}{\sqrt{\left(1+a^{2}\right)}}, \quad C V\left(1+a^{2}\right), \quad \frac{a C}{\sqrt{\left(1+a^{2}\right)}}, \quad a C V\left(1+a^{2}\right)$, $\frac{C}{a V\left(1+a^{2}\right)}, \frac{C V\left(1+a^{2}\right)}{a}$ untersucht. Sie baben aber entweder nur zu den bereits erhaltenen Resultaten, oder zu imaginären Flächen, oder zu der Umdrehungsfliche der Kettenlinie geführt; oder die Elimination liefs sich nicht bewerkstelligen; weswegen ich es für unnöthig halte, sie einzeln hier durchzugehen.

Zu der Schrạubenfläche (6.) und der Umdrehungsfläche der Kettenlinie (8.) sind also gegenwärtig noch fünf, durch die Gleichungen (7.), 
202 10. Scherk, Bemerkungen über die kleinste Fläche innerhalb gegebener Grenzen.

(9.), (16.), (20.), (30.) dargestelite, neue Flächen hinzugekommen, deren erste ich, in ihrer speciellen Form (6.), im Verein mit der letzten, in einer bald folgenden Abhandlung besonders untersuchen werde, und deren zweite, wie bereits erwähnt, die merkwürdige Eigenschaft hat, dafs zwei, in Entstehungsart und Form sich auf das Bestimmteste von einander unterscheidende, Flächen als specielle Fälle von ihr erscheinen.

\section{9.}

Wir gehen nun zu der angekündigten zweiten Untersuchung über, durch welche wir zu entscheiden suchen, welche Form die willkürlichen Functionen in dem Integrale (12.) haben müssen, wenn die kleinste Fläche durch Bewegung einer geraden Linie entstanden sein soll. Hierbei werden wir so zu Werke gehen, dafs wir zuerst aus den Gleichungen (12.) die Werthe von $p, q, r, s, t$ herleiten werden, die der Gleichung (1.) der kleinsten Fläche angehören; diese Werthe werden wir alsdann in die allgemeine Gleichung der durch Bewogung einer geraden Linie entstandenen Fläche substituiren, und uns auf diese Weise eine neue Gleichung verschaffen, die beiden genannten Flächen angehört; die Integration derselben führt dann zu der gewünschten Form der willkürlichen Functionen.

Durch Differentiation des Systems der Gleichungen (12.) hat man nämlich :

$$
\text { 36. } \quad\left\{\begin{array}{l}
d x=i A^{\prime} d a+i B^{\prime} a b, \\
d y=-i a A^{\prime} d a-i b B^{\prime} d b, \\
d z=-\sqrt{ }\left(1+a^{2}\right) A^{\prime} d a+\sqrt{ }\left(1+b^{2}\right) B^{\prime} d b .
\end{array}\right.
$$

Da aber $z$ eine Function von $x$ und $y$ ist, so hat man auch

$$
d z=p d x+q d y=i A^{\prime}(p-q a) d a+i B^{\prime}(p-q b) d b,
$$

welches, mit dem obigen Werthe von $d z$ verglichen,

also

$$
\text { 37. }\left\{\begin{array}{l}
p-q a-i v\left(1+a^{2}\right)=0, \\
p-q b+i v\left(1+b^{2}\right)=0
\end{array}\right.
$$

giebt. Ferner ist

$$
\text { 38. }\left\{\begin{array}{l}
p=\frac{i b V\left(1+a^{2}\right)+i a V\left(1+b^{2}\right)}{b-a}, \\
q=\frac{i V\left(1+a^{2}\right)+i V\left(1+b^{2}\right)}{b-a}
\end{array}\right.
$$

$$
\begin{aligned}
& d p=r d x+s d y=i A^{\prime}(r-s a) d a+i B^{\prime}(r-s b) d b \\
& d q=s d x+t d y=i A^{\prime}(s-t a) d a+i B^{\prime}(s-t b) d b
\end{aligned}
$$

also 
10. Scherk, Bemerkungen über die kleinste Fläohe innerhalb gegebener Grenzen. 203

$$
\begin{array}{ll}
\left(\frac{d p}{d a}\right)=i A^{\prime}(r-s a), & \left(\frac{d q}{d a}\right)=i A^{\prime}(s-t a), \\
\left(\frac{d p}{d b}\right)=i B^{\prime}(r-s b), & \left(\frac{d q}{d b}\right)=i B^{\prime}(s-t b) .
\end{array}
$$

Vermittelst dieser Werthe erhält man, durch Differentiation der Gleichungen (37.) in Beziehung auf $a$ und $b$ :

$$
\begin{aligned}
& i A^{\prime}(r-s a)-i A^{\prime}(s-t a) a-q-\frac{i a}{\sqrt{\left(1+a^{2}\right)}}=0, \\
& \quad r-s b-(s-t b) a=0, \\
& i B^{\prime}(r-s b)-i B^{\prime}(s-t b) b-q+\frac{i b}{\sqrt{\left(1+b^{2}\right)}}=0,
\end{aligned}
$$

d. h., wenn für $q$ gein Werth gesetzt wird:

wo Kürze halber

$$
\text { 39. }\left\{\begin{aligned}
r-2 a s+a^{2} t & =\frac{C}{a^{2}}, \\
r-(a+b) s+a b t & =0, \\
r-2 b s+b^{2} t & =\frac{C}{\beta^{2}},
\end{aligned}\right.
$$

$$
\text { 40. } \quad\left\{\begin{array}{r}
\frac{1+a b+V\left(1+a^{2}\right) V\left(1+b^{2}\right)}{b-a}=C, \\
r\left(1+a^{2}\right) \cdot A^{\prime}=a^{2}, \\
r\left(1+b^{2}\right) \cdot B^{\prime}=\beta^{2}
\end{array}\right.
$$

gesetzt worden ist. Multiplicirt man nun die drei Gleichungen (39.) resp. durch

und addirt, so erhält man

$$
\begin{aligned}
& b^{2},-2 a b,+a^{2}, \\
& \text { alsdann durch } b,-(a+b), \quad a, \\
& \text { endlich durch } 1,-2,+1,
\end{aligned}
$$

$$
41 * \quad\left\{\begin{array}{l}
(b-a)^{2} r=\frac{b^{2} C}{\alpha^{2}}+\frac{a^{2} C}{\beta^{2}}, \\
(b-a)^{2} s=\frac{b C}{\alpha^{2}}+\frac{a C}{\beta^{2}}, \\
(b-a)^{2} t=\frac{C}{a^{2}}+\frac{C}{\beta^{2}} .
\end{array}\right.
$$

*) Beiläufig mag bemerkt werden, wie man vermittelst (38.) und (41.) selhr leicht zeigen kann, dafs die Gleichungen (12.) das Integral von $\left(1+q^{2}\right) r-2 p q s$ $+\left(1+p^{2}\right) t=0$ darstellen. Denn bezeichnet man die Zähler von $p$ nnd $q$ in (38.) durch $p^{\prime}$ und $q^{\prime}$, und setzt die Resultate aus (38.) und (41.) in den ersten Theil der eben genannten Gleichung, so wird derselbe $=$

$h=\frac{C}{(b-a)^{4}}\left\{\frac{1}{a^{2}}\left[\left(1+b^{2}\right)(b-a)^{2}+\left(p^{\prime}-b q^{\prime}\right)^{2}\right]+\frac{1}{\beta^{2}}\left[\left(1+a^{2}\right)(b-a)^{2}+\left(p^{\prime}-a q^{\prime}\right)^{2}\right]\right\}$. 
204 10. Scherk, Bemerkungen über die kleinste Fläche innerhalb gegelener Grenzen.

Setzt man ferner

$$
\begin{aligned}
& d r=\lambda d x+\mu d y=i A^{\prime}\left(\lambda-\mu_{a} a\right) d a+i B^{\prime}(\lambda-\mu b) d b, \\
& d s=\mu d x+\nu d y=i A^{\prime}(\mu-\nu a) d a+i B^{\prime}(\mu-\nu b) d b, \\
& d t=\nu d x+\omega d y=i A^{\prime}(\nu-\omega a) d a+i B^{\prime}(\nu-\omega b) d b,
\end{aligned}
$$

differentiirt die Gleichungen (39.), und zwar die erste in Beziehung auf $a$, die zweite auf $a$ und auf $b$, die dritte auf $b$, bemerkt, dafs

$$
\left(\frac{d C}{d a}\right)=\frac{V\left(1+b^{2}\right)}{(b-a)^{2}}\left[\frac{1+a b}{V\left(1+a^{2}\right)}+r\left(1+b^{2}\right)\right]=\frac{V\left(1+b^{2}\right)}{V\left(1+a^{2}\right)} \cdot \frac{C}{b-a}=\frac{\beta^{2} A^{\prime}}{\alpha^{2} B^{\prime}} \cdot \frac{C}{b-a},
$$

und auf gleiche Weise

$$
\left(\frac{d C}{d b}\right)=-\frac{V\left(1+a^{2}\right)}{V\left(1+b^{2}\right)} \cdot \frac{C}{b-a}=-\frac{\alpha^{2} B^{\prime}}{\beta^{2} A^{\prime}} \cdot \frac{C}{b-a},
$$

und, dafs endlich $s-a t=\frac{c}{(b-a) \alpha^{2}}, s-b t=-\frac{c}{(b-a) \beta^{2}}$ : so erhält man, nach einfachen Reductionen:

42.

$$
\left\{\begin{array}{l}
\lambda-3 a \mu+3 a^{2} \nu-a^{3} \omega=\frac{C i}{\alpha^{2}}\left[\frac{2}{(b-a) A^{\prime}}+\frac{\beta^{2}}{(b-a) a^{2} B^{\prime}}-\frac{2 d a}{\alpha A^{\prime} d a}\right] \\
\lambda-(2 a+b) \mu+\left(a^{2}+2 a b\right) \nu-a^{2} b \omega=-\frac{C i}{A^{\prime}(b-a) \beta^{2}}, \\
\lambda-(a+2 b) \mu+\left(2 a b+b^{2}\right) \nu-a b^{2} \omega=-\frac{C i}{B^{\prime}(b-a) \beta^{2}}, \\
\lambda-3 b \mu+3 b^{2} \nu-b^{3} \omega=\frac{C i}{\beta^{2}}\left[\frac{2}{(b-a) B^{\prime}}+\frac{\alpha^{2}}{(b-a) \beta^{2} A^{\prime}}+\frac{2 d \beta}{\beta B^{\prime} d b} .\right.
\end{array}\right.
$$

Setzt man die zweiten Theile dieser Gleichungen Kürze halber resp.

$$
=-C i P, \quad C i Q,-C i R, C i S
$$

und multiplicirt sie resp. durch *)

Da sun

$$
\begin{aligned}
& p^{\prime}-b q^{\prime}=-i(b-a) \sqrt{ }\left(1+b^{2}\right), \\
& p^{\prime}-a q^{\prime}=+i(b-a) \sqrt{ }\left(1+a^{2}\right)
\end{aligned}
$$

so ist der Facior von $\frac{1}{\alpha^{2}}$ sowohl, als der von $\frac{1}{\beta^{2}}$, besonders $=0$, also $h=0$, wie gehörig.

*) Diese und die oben angeführten Multiplicatoren der Gleichungen (39.) befolgen das Gesetz, dafs die in der obersten Horizontalreihe stehenden, die Glieder der Binomialreihe sind, und dafs aus jedem Factor von der Form $\Sigma k_{.} a^{p} b^{q}$, wo $k$ der numerische Coefficient ist, der darunter stehende Factor $=\Sigma \frac{k}{p+q}\left(p a^{p-1} b^{q}+q a^{p} b^{q-1}\right)$ gebildet wird. Dals dieselbe Bildungsweise bei der Bestimınung der fünf partiellen Differentialquotienten der vierten Ordnung, der sechs der fünften Ordnung u. s. f: angewandt werden kann, übersieht man leicht, wenn man untersucht, auf welche Weise die auf einander folgenden Potenzen vou $b-a$ aus den so gebildeten MIultiplicatoren und den Coefficienten resp. von $p, q ; r, s, t ; \lambda, \mu, \nu, \omega$ in (37.), (39.), (42.) zusammengesetzt sind. 
alsdann durch

$$
b^{3}, \quad-3 a b^{2}, \quad+3 a^{2} b, \quad-a^{3},
$$

hierauf durch

$$
b^{2},-\left(b^{2}+2 a b\right), 2 a b+a^{2},-a^{2},
$$

endlich durch

$$
b, \quad-(2 b+a), \quad 2 a+b, \quad-a,
$$

so ergiebt sich

$$
\text { 1, } \quad-3, \quad 3, \quad-1 \text {, }
$$

$$
\left\{\begin{array}{l}
\frac{(b-a)^{3} \lambda i}{c}=\lambda^{\prime}=b^{3} P+3 a b^{2} Q+3 a^{2} b R+a^{3} S, \\
\frac{(b-a)^{3} \mu i}{C}=\mu^{\prime}=b^{2} P+\left(b^{2}+2 a b\right) Q+\left(2 a b+a^{2}\right) R+a^{2} S, \\
\frac{(b-a)^{3} \nu i}{C}=\nu^{\prime}=b P+(2 b+a) Q+(2 a+b) R+a S, \\
\frac{(b-a)^{3} \omega i}{C}=\omega^{\prime}=P+3 Q+3 R+S .
\end{array}\right.
$$

Durch diese Gleichungen werden also die vier Differentialquotienten der dritten Ordnung $\lambda$, $\mu, \nu, \omega$, die der kleinsten Fläche angehören, bestimmt. Nun ist bekanntlich die Gleichung jeder durch Bewegung einer geraden Linie entstandenen Fläche:

$$
\text { 44. } \lambda+3 \mu m+3 \nu m^{2}+\omega m^{3}=0 \text {, }
$$

aus welcher $m$ vermittelst der Gleichung

$$
\text { 45. } r+2 s m+t m^{2}=0
$$

eliminirt werden mufs. Soll also die kleinste Fläche durch Bewegung einer geraden Linie entstanden sein, so müssen die Werthe von $\lambda, \mu$, $v$, $\omega$, oder, was dasselbe ist, von $\lambda^{\prime}, \mu^{\prime}, \nu^{\prime}, \omega^{\prime}$, der Gleichung (44.) Genüge leisten. Werden sie aber aus (43.) in (44.) substituirt, so erhält man $(b+m)^{3} P+3(b+m)^{2}(a+m) Q+3(b+m)(a+m)^{2} R+(a+m)^{3} S=0$, also, wenn

gesetzt wird:

$$
\frac{a+m}{b+m}=n, \text { und daher } m=-\left(\frac{a-b n}{1-n}\right)
$$

46. $P+3 Q n+3 R n^{2}+S^{3} n=0$,

und die Gleichung (38.) geht hierdurch in folgende über:

$$
r-2 a s+t a^{2}-2(r-(a+b) s+a b t) n+\left(r-2 b s+b^{2} t\right) n^{2}=0 \text {, }
$$

in welcher die Coefficienten der einzelnen Potenzen von $n$ die ersten Glieder von (39.) sind. Folglich hat man

$$
\begin{gathered}
\frac{1}{\alpha^{2}}+\frac{n^{2}}{\beta^{2}}=0 \\
n=\frac{\beta i}{\alpha} .
\end{gathered}
$$


206 10. Scherk, Bemerkungen über die kleinste Fläche innerhalb gegebener Grenzen.

Hierdurch wird nun (46.)

$$
p \alpha^{3}+3 Q \alpha \beta^{3} i-3 R \alpha \beta^{2}-S \beta^{3} i=0 \text {. }
$$

Setzt man hierin für $P, Q, R, S$, ihre Werthe aus (42.), so ergiebt sich:

$$
\frac{1}{b-a}(\beta+\alpha i)\left(\frac{\alpha}{\beta A^{\prime}}-\frac{\beta}{\alpha B^{\prime}}\right)-\frac{d \cdot a}{A^{\prime} d a}-\frac{i d \beta}{B^{\prime} d b}=0 \text {; }
$$

oder, da $\frac{\alpha}{\beta A^{\prime}}-\frac{\beta}{\alpha B^{\prime}}=\frac{V\left(1+a^{2}\right)-V\left(1+b^{2}\right)}{\alpha \beta}$, so ist

$$
\text { 47. }\left(\frac{V\left(1+a^{2}\right)-V\left(1+b^{2}\right)}{b-a}\right)\left[\frac{1}{\alpha}+\frac{i}{\beta}\right]-\frac{d a}{A^{\prime} d a}-\frac{d \beta}{B^{\prime} d b}=0 \text {, }
$$

welcher Gleichung man folgende Form geben kann:

$$
\begin{aligned}
& \text { 48. } \quad\left[\frac{V\left(1+a^{2}\right)}{\alpha}+\frac{a V\left(1+a^{2}\right)}{a^{2}} \cdot \frac{d \alpha}{d a}\right]-\left[\frac{V\left(1+b^{2}\right)}{\beta}+\frac{b V\left(1+b^{2}\right)}{\beta^{2}} \cdot \frac{d \beta}{d b i}\right] i \\
& -\frac{V\left(1+b^{2}\right)}{\alpha}+\frac{i V\left(1+a^{2}\right)}{\beta}-\frac{b V\left(1+a^{2}\right)}{a^{2}} \cdot \frac{d \alpha}{d a}+\frac{a i V\left(1+b^{2}\right)}{\beta^{2}} \cdot \frac{d \beta}{d b}=0 .
\end{aligned}
$$

Durch diese Gleichung werden hiernach die beiden willkürlichen Functionen $A, B$, vermittelst der mit ihnen durch die Gleichungen (40.) verbundenen willkürlichen Functionen $\alpha, \beta$, bestimmt. Nun bemerke man aber, dafs (48.) aus 3 sehr wesentlich verschiedenen Gattungen von Gliedern besteht, von denen die erste von $a$ allein, die zweite von $b$ allein, und die dritte von $a$ und $b$ zugleich abhängig ist. Da aber $a$ und $b$ von einander unabbängige Quantitäten sind, so kann jener Gleichung auf keine andere Art Grt Genüge geleistet werden, als dadurch, dafs man

$$
\text { 49. }\left\{\begin{array}{l}
\frac{V\left(1+a^{2}\right)}{a}+\frac{a V\left(1+a^{2}\right)}{a^{2}} \cdot \frac{d a}{d a}=F, \\
\frac{V\left(1+b^{2}\right)}{\beta}+\frac{b V\left(1+b^{2}\right)}{\beta^{2}} \cdot \frac{d \beta}{d b}=-F i
\end{array}\right.
$$

setzt, wo $F$ eine beliebige Constante ist, und dafs man diese, so wie die beiden durch die Integration der Gleichungen (49.) in die Rechnung kommenden Constanten, so bestimmt, dafs aufserdem, wenn dies möglich ist, noch der Gleichung

50.

$$
-\frac{V\left(1+b^{2}\right)}{\alpha}+\frac{i V\left(1+a^{2}\right)}{\beta}-\frac{b V\left(1+a^{2}\right)}{a^{2}} \cdot \frac{d \alpha}{d a}+\frac{a i V\left(1+b^{2}\right)}{\beta^{2}} \cdot \frac{d \beta}{d b}=0
$$

Genige geleistet werde. Die vollständigen Integrale von (49.) sind aber

$$
\begin{aligned}
& \frac{1}{a}=E a+F \sqrt{ }\left(1+a^{2}\right), \\
& \frac{1}{\beta}=E^{\prime} b-F i \checkmark\left(1+b^{2}\right),
\end{aligned}
$$

wo $E, E^{\prime}$ die Constanten der Integration siw l. Hierdurch wird der erste Theil von (50.) 


$$
\left(E+E^{\prime} i\right)\left(b \sqrt{ }\left(1+a^{2}\right)-a \sqrt{ }\left(1+b^{2}\right)\right),
$$

und demnach wird jener Gleichung Genüge geleistet, indem man

$$
\boldsymbol{E}^{\prime}=\boldsymbol{E} \boldsymbol{i}
$$

setzt, woraus

$$
\text { 51. }\left\{\begin{array}{l}
\frac{1}{a}=E a+F r\left(1+a^{2}\right), \\
\frac{1}{\beta}=i\left(E b-F \checkmark\left(1+b^{2}\right)\right.
\end{array}\right.
$$

folgt, wo $E, F$ beliebige Constanten bezeichnen. Durch diese Gleichung ist jedoch noch nicht das vollständige Integral von (48.) bestinimt. Denn man kann ihr, wie aus der Form (4\%) erhellt, auch noch auf eine particuläre Art Genüge leisten, indem man

also

$$
\frac{1}{\alpha}+\frac{i}{\beta}=0
$$

$$
\frac{1}{\alpha}=+D, \quad \frac{1}{\beta}=-D
$$

setzt, wo $D$ eine neue Constante ist; und wenn man diese beiden Integrale in Eins vereinigt, so, dals

$$
\begin{aligned}
& \frac{1}{a}=D+E a+F r\left(1+a^{2}\right), \\
& \frac{1}{\beta}=i\left(D+E b-F \vee\left(1+b^{2}\right)\right)
\end{aligned}
$$

wird, so hat man die allgemeinen Formen erhalten, welche den willkürlichen Functionen $\alpha, \beta$ beigelegt werden müssen, wenn die kleinste Fläche durch Bewegung einer geraden Linie entstanden sein soll. Setzt man diese Werthe in (40.), so erhält man $A^{\prime}, B^{\prime}$, und, vermittelst (12.), sodann $x, y, z$ ausgedrückt durch $a$ und $b$. Der Integration steht kein Hindernifs im Wege, wohl aber der Elimination, so lange man nicht den Constanten specielle Werthe beilegt, welche die Rechnung vereinfachen. Die Systeme specieller Werthe aber, die sich zunächst darbieten, sind

$$
\begin{array}{ll}
\boldsymbol{E}=0, & \boldsymbol{F}=0, \\
\boldsymbol{F}=0, & \boldsymbol{D}=0, \\
\boldsymbol{D}=0, & \boldsymbol{E}=0 .
\end{array}
$$

Im ersten Falle hat $\operatorname{man} \frac{1}{\alpha^{\prime}}=-\frac{i}{\beta}, \frac{1}{\alpha^{2}}+\frac{1}{\beta^{2}}=0$, folglich, vermöge (41.), $t=0$, welche Gleichung nur auf die Schraubenfläche führt. (S. meine angef. Abh. pag. 225.) 
208 10. Scherk, Bemerkungen über die Kleinste Fläche innerhalb gegebener Grenzen.

Im zweiten Falle hat $\operatorname{man} \frac{1}{a c}=\frac{i}{b \beta}$, also $\frac{b^{2}}{a^{2}}+\frac{a^{2}}{\beta^{2}}$, und folglich, vermöge derselben Gleichung (41.), $r=0$, in welcher Gleichung abermals nur die Schraubenflïche enthalten ist.

Im dritten Falle ist $\frac{1}{\sqrt{\left(1+a^{2}\right) \cdot a}}=-\frac{i}{\sqrt{\left(1+b^{2}\right) \cdot \beta}}$, folglich $\frac{1+b^{2}}{a^{2}}+$ $\frac{1+a^{2}}{\beta^{2}}=0$. Hierdurch wird aber $r+t=0$, und auch diese Gleichung (ührt nur auf die Schraubenflïche (a. a. O. pag. 234). Es haben also die einzelnen Theile unserer für $\frac{1}{\alpha}, \frac{1}{\beta}$ gefundenen Ausdrücke die eigenthümliche Eigənschaft, dals jeder von ihnen, für sich genommen, auf dieselbe Fläche führt, was, in Verbindung mit dem Umstande, dafs in der Gleichung der durch 2 grade Linien begrenzten kleirsten Fläche, wenn sie auf ihre einfachste Gestalt gebracht und von der Lage der Coordinaten unabhängig gemacht wird, nur eine Constante vorkommen kann, weil sie von 2 andern geraden Linien sich nur durch ihren Abstand unterscheiden, dafs also von einer Relation verschiedener Constanten, welche bewirken künnte, dafs die genannte kleinste Fläche verschiedene Gestalten haben könnte, nicht die Rede sein kann, es im höchsten Grade wahrscheinlich macht, dafs ron allen Flächen nur die Schraubenflïche durch Bewegung einer geraden Linie entstanden und zugleich die kleinste zwischen gegchenen Grenzen sein kann, während es allerdings noch ganz andere Gleichungen zwischen $x, y$, als die Gleichung der Schraubenflïche giebt, die zugleich den Gleichungen (1.), (44.) und (45.) Genüge leisten.

Zuletzt mag der Vortheil nicht unerwähnt bleiben, der für die Rechnung durch Einführung der Functionen $\alpha, \beta$ statt der in dem Integrale (4.) vorkommenden $\varphi a, \psi b$, erwachsen ist. Denn, während wir z. B. im gegenwärtigen Falle $\alpha$ und $\beta$ nur constant zu setzen brauchten, um die Schraubenfläche zu erzeugen, mufs $\varphi a=\frac{i \cos n a}{n}, \psi b=\frac{i \cos n b}{n}$ angenommen werden, um zu derselben Fläche zu gelangen.

Kiel, im Juli 1834 . 